\title{
MODELO DE IMPLEMENTACIÓN DE APRENDIZAJE SERVICIO (A+S) EN LA UC. UNA EXPERIENCIA QUE IMPACTA POSITIVAMENTE EN LA FORMACIÓN PROFESIONAL INTEGRAL
}

\section{Introducción}

Sin duda el proceso de formación profesional debiera impactar en el aprendizaje de los estudiantes, quienes una vez terminada su carrera, tendrían que estar en condiciones de aportar a la sociedad una serie de destrezas, conocimientos, valores y actitudes. Hoy, no basta solo con entregar conocimientos, sino que se requiere que los estudiantes aprendan herramientas que les permitan resolver problemas en escenarios de alta complejidad y renovación de los saberes.

Para ello, no solo es necesario contar con docentes expertos en sus materias, sino también con profesores que sepan cómo hacer que sus alumnos dominen estos conocimientos y las formas más apropiadas para que puedan ponerlos en práctica de manera responsable, considerando la realidad social actual. En este sentido, es necesario apostar por la formación de profesionales que desarrollen habilidades para construir un conocimiento que les sea útil y de la forma más significativa posible, para tomar decisiones en los diferentes escenarios de su campo profesional. Sin embargo, también se debe apostar por la formación de ciudadanos que ejerzan su profesión al servicio de la sociedad.

En este contexto, la metodología de Aprendizaje Servicio se presenta como una herramienta que permitiría a las instituciones de educación superior responder integradamente a tres dimensiones: una académica, donde el contacto directo con la realidad posibilitaría mayores y mejores aprendizajes en los estudiantes; otra ligada con la calidad del servicio y la contribución real a la solución de la problemática comunitaria; y finalmente, la posibilidad de generar un espacio de formación en valores para los estudiantes. 
El presente artículo desarrolla las principales características del modelo de incorporación de la metodología A+S en la Pontificia Universidad Católica y los principales resultados en sus nueve años de implementación.

\section{Antecedentes teóricos y contextuales}

Existen diferentes tipos de experiencias educacionales que vinculan el servicio a la comunidad con la educación formal, pero no todas estas experiencias pueden ser denominadas Aprendizaje Servicio (A+S). El aspecto clave para considerar a una experiencia educacional como $\mathrm{A}+\mathrm{S}$ es el equilibrio entre los aprendizajes de los estudiantes con el servicio orientado a una necesidad real de una comunidad (Furco y Billing, 2002). De esta manera, las iniciativas universitarias que se enfocan en entregar un servicio solidario, como voluntariados y campañas, no podrían ser consideradas $A+S$, ya que no tienen por foco el aprendizaje de los estudiantes, sino que el servicio solidario ofrecido. Las prácticas profesionales tradicionales tampoco cabrían dentro de la definición de $\mathrm{A}+\mathrm{S}$, ya que la atención de la metodología no es únicamente el desarrollo de la experiencia profesional o la preparación para el mundo laboral, sino que además el desarrollo del compromiso social y de un énfasis en la experiencia de servicio.

Precisamente el equilibrio entre la necesidad comunitaria y los objetivos de aprendizaje es lo que se destaca en las definiciones de A+S disponibles en la literatura. Furco y Billing (2002) definen el Aprendizaje Servicio como una metodología pedagógica experiencial, que se caracteriza por la integración de actividades de servicio a la comunidad en el currículo académico, donde los alumnos utilizan los contenidos y las herramientas académicas en atención a necesidades genuinas de una comunidad. Tapia (2006), por su parte, considera el $\mathrm{A}+\mathrm{S}$ como una filosofía educativa, que subraya el valor de las actividades solidarias al servicio de la comunidad y desarrolla su potencial educativo, conectándolas con el aprendizaje formal. Eyler (2009), en tanto, define el Aprendizaje Servicio como una forma de educación experiencial que combina el estudio académico con el servicio a la comunidad, mejorando la calidad de ambos y que apunta a un cambio en el paradigma educativo, hacia un enfoque participativo y democrático. 
La integración de los objetivos pedagógicos y de servicio se realiza mediante un diseño del curso en donde la reflexión tiene un rol central. De este modo, los tres elementos centrales de la metodología son el aprendizaje, el servicio y la reflexión estructurada (Hansen, 2012; Eyler, 2009). La estrategia de reflexión en A+S es entendida como el diseño de espacios en el curso antes, durante y después de la experiencia de servicio, que apunten a que los estudiantes logren una comprensión profunda de los aspectos históricos, sociológicos, culturales, económicos y políticos que están detrás de las necesidades de la comunidad (Cooper, Cripps y Reisman, 2013). Asimismo, en los espacios de reflexión los estudiantes tienen la posibilidad de significar su práctica en la comunidad, relacionar los contenidos del curso con la experiencia de servicio, formular preguntas, proponer teorías y planes de acción y expresar sus ideas, lo que es crítico para la consolidación de aprendizajes (Eyler, 2001).

La investigación muestra que el A+S es una metodología que fomenta la adquisición de conocimientos, habilidades, actitudes y valores en estudiantes de educación superior. Por ejemplo, Jenkins y Sheehey (2011) plantean que esta metodología aumenta la adquisición de conocimientos disciplinares, a la vez que fomenta la confianza y el compromiso social en los estudiantes. Groh, Stallwood y Daniels (2011) complementan afirmando que por medio de la metodología se desarrollan el liderazgo y valores como la justicia social. Cooper, Cripps y Reisman (2013) evidencian que la experiencia de Aprendizaje Servicio impacta positivamente en los estudiantes en cuanto a la percepción de su rol como agentes de cambio de la sociedad, encontrando además una sólida correlación entre las experiencias de $\mathrm{A}+\mathrm{S}$ y el compromiso cívico y las actitudes altruistas en los estudiantes. En un estudio longitudinal realizado a exalumnos que tuvieron $\mathrm{A}+\mathrm{S}$ a lo largo de su carrera, se evidenció que la metodología influyó en sus elecciones profesionales, haciendo que muchos tendieran a optar por trabajos que sirvieran a la comunidad, así como también que dicha experiencia desarrolló sus habilidades de comunicación, liderazgo, trabajo en equipo y emprendimiento, además de las habilidades para asesorar y facilitar grupos (Newman y Hernández, 2011). 
Por otra parte, existen dos grandes aproximaciones para la implementación de la metodología $\mathrm{A}+\mathrm{S}$ en la universidad: una es de forma curricular y la otra es cocurricularmente. En el primer caso, la metodología se inserta dentro de un currículo profesional determinado, por lo que la actividad de servicio se conecta directamente con las habilidades profesionales. En el segundo caso -la implementación cocurricular- la metodología se inserta como una actividad de extensión de la universidad, o bien, como un curso optativo multidisciplinario en el que se aplica la metodología (Howard, 2003; Jenkins y Sheehey, 2011).

En la Pontificia Universidad Católica de Chile se ha optado por un modelo de implementación de $\mathrm{A}+\mathrm{S}$ de corte curricular, de manera de poder responder a la formación disciplinaria de cada perfil profesional, según la Impronta del egresado UC y el proyecto educativo, de forma coherente con los valores y misión de la universidad, los que aspiran a lograr la excelencia en la creación y transferencia del conocimiento y en la formación de personas y que está inspirada en una concepción católica y siempre al servicio de la Iglesia y la sociedad (Pontificia Universidad Católica de Chile, 2010). De esta manera, los objetivos de la inserción de la metodología en la universidad son:

- Desarrollar una docencia que genere aprendizajes significativos basados en el vínculo con la sociedad.

- Aportar al desarrollo de las comunidades por medio de los proyectos realizados por los estudiantes de la UC en el contexto de sus actividades académicas.

- Potenciar en los estudiantes el desarrollo del compromiso social y la formación en valores.

\section{Antecedentes históricos}

El A+S comenzó a incorporarse en la UC a partir de la inquietud de un grupo de académicos, quienes en el año 2004 plantean la necesidad de organizar un grupo de prácticas de servicio que se realizaban en varios cursos de la universidad. De esta forma, ese año se instaló el Programa A+S en la Dirección de Asuntos Estudiantiles. En 2005 se decidió trasladar el programa al Centro de Desarrollo Docente 
(CDDoc), dependiente de la Vicerrectoría Académica, de modo de facilitar la inserción curricular de la metodología, así como también aportar a los procesos de formación y acompañamiento que los docentes requieren para la implementación de los cursos.

De esta manera, uno de los ejes centrales del programa A+S en la UC se caracteriza por la capacitación y el acompañamiento de los profesores en la implementación de esta metodología en sus cursos.

La metodología Aprendizaje Servicio ha sido implementada en la UC principalmente en los cursos obligatorios de las diferentes carreras de pregrado. Las experiencias tienen por lo general una duración semestral, como lo son casi todos los cursos de la universidad, aun cuando existen algunos casos en los que los ramos o los docentes continúan su relación con un socio comunitario.

Los servicios ofrecidos por los estudiantes son, por lo general, asesorías en temas específicos, los que contemplan también diagnósticos y retroalimentación; capacitación en ciertos temas de utilidad para los socios; elaboración de herramientas materiales e intangibles; y levantamiento de información relevante para las instituciones.

Es así, por ejemplo, que desde 2005 los estudiantes de quinto año de la carrera de Psicología en el ramo Gestión de Personas han desarrollado sistemas de selección y reclutamiento de voluntarios para diversas fundaciones, han creado perfiles de cargo y evaluaciones de desempeño para organizaciones no gubernamentales y han asesorado cambios organizacionales. La calificación de los estudiantes en el ramo recae en la calidad del servicio que son capaces de prestar a las instituciones socias, utilizando para ello los contenidos y las herramientas que el curso pone a su disposición. Del mismo modo, los estudiantes de la carrera de Química en su primer semestre consolidan lo aprendido en el curso Química en la naturaleza y la sociedad, realizando talleres prácticos de laboratorio a niños de educación básica, participantes de un programa de promoción de la ciencia, patrocinado por la I. Municipalidad de La Pintana. 


\section{Modelo de incorporación de la metodología}

Desde 2005 la UC ha formalizado un modelo de incorporación de la metodología, el que presenta las siguientes características:

\section{a. Características pedagógicas del modelo}

El modelo tiene un acento curricular en donde el servicio está ligado a los objetivos de aprendizaje de cada curso y carrera. Gran parte de la labor de los profesionales del programa A+S UC es, precisamente, la capacitación y asesoría a los equipos docentes para que se realice un diseño óptimo de las experiencias, que liguen de manera coherente objetivos de aprendizaje (cognitivos, procedimentales y valóricos) y de servicio de los diferentes cursos. Para ello, cada docente analiza un problema social o comunitario en el que su disciplina podría ser un aporte, verifica la posibilidad de efectuar un servicio para responder a ese problema y evalúa la coherencia de la incorporación de dicho servicio con el logro de los objetivos de aprendizaje.

Además, la metodología del Aprendizaje Servicio es implementada principalmente en cursos obligatorios de las diferentes carreras de pregrado, en donde las experiencias tienen por lo general una duración semestral. Por otra parte, la decisión de implementar un curso con la metodología $A+S$ es voluntaria, por lo que si los docentes deciden incorporar esta metodología, solicitan la asesoría del Programa para las primeras implementaciones.

El modelo presenta también la incorporación de la reflexión estructurada como herramienta pedagógica, la que representa una instancia que planifica el docente y que implementa durante la clase, con el objetivo principal de unir el servicio con el aprendizaje. Los temas en cada curso varían, pudiendo tratarse principalmente de un análisis respecto de la comunidad intervenida, la evaluación de la experiencia vivida, $u$ otras habilidades y/o valores planificados como objetivos de aprendizaje por el docente. Ejemplo de ello podrían ser el compromiso social y el trabajo en equipo, entre otros.

El modelo incluye, además, una evaluación final de estudiantes y socios comunitarios realizada por una plataforma en línea. El 
objetivo principal de esta evaluación es retroalimentar al profesor en la incorporación de $\mathrm{A}+\mathrm{S}$ y permitir también la recogida de datos acerca de las características del aprendizaje que evidencian los estudiantes en estos cursos.

\section{b. Características de la implementación de A+S en los cursos}

La incorporación de la metodología está estructurada sobre la base de un conjunto de procesos que se acompaña por un programa de capacitación y una asesoría pedagógica, elementos que permitirían asegurar la calidad de las implementaciones.

El modelo presenta igualmente hitos esenciales antes, durante y una vez terminado el curso (Figura 1):

- Evaluación de la factibilidad para realizar un servicio en el curso: se revisa aquí la coherencia de los objetivos de aprendizaje con un servicio a la comunidad, la carga de trabajo del estudiante y el posible socio comunitario, entre otros elementos.

- Planificación inicial del curso: en esta etapa el docente adecúa la planificación de su curso para incorporar una actividad de servicio, así como también diseña reflexiones y evaluaciones para el mismo.

- Constitución Sociedad A+S: en esta instancia se verifica la búsqueda y el contacto con los socios comunitarios, firmando un documento de acuerdo para planificar y consensuar los términos del servicio.

- Presentación inicial A+S en cursos: en este momento el docente explica al curso la metodología y sus impactos en el aprendizaje y en la comunidad.

- Evaluación temprana: se define un espacio para evaluar e incorporar las mejoras en la implementación del curso y del servicio.

- Encuesta final a los estudiantes y a los socios.

- Autoevaluación final compartida. 
Figura 1: Modelo de implementación A+S

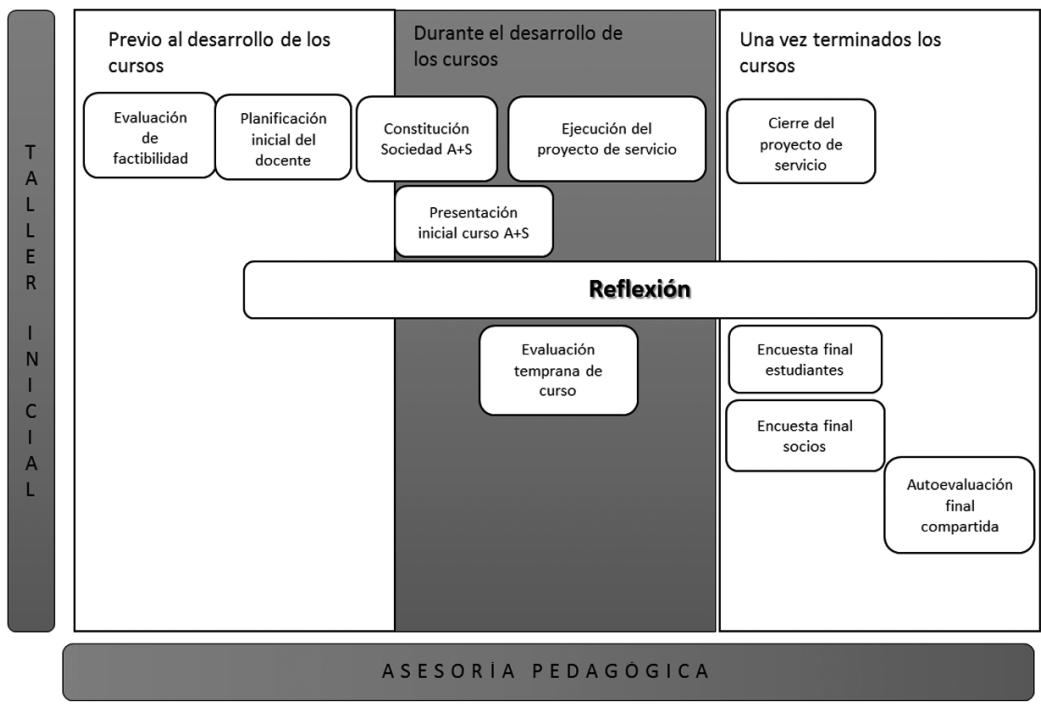

El modelo A+S busca que el académico conozca y haga propia la metodología, de modo de ser capaz de introducir adecuaciones a sus cursos, por lo que es central la capacitación y la asesoría docente. Al respecto, la capacitación docente consiste en una serie de talleres diseñados especialmente para que estos se familiaricen con la metodología y adquieran herramientas pedagógicas básicas para el diseño de cursos con A+S. La asesoría, en tanto, implica el acompañamiento al docente para que el curso evidencie objetivos de aprendizaje y de servicio a la comunidad de forma coherente, y que sean a su vez pertinentes, alcanzables y posibles de evaluar durante el tiempo de desarrollo de un curso. La asesoría incluye, además, el apoyo para que el docente diseñe espacios de reflexión estructurados antes, durante y al finalizar la actividad de servicio, con el propósito de alcanzar aprendizajes profundos y significativos en los estudiantes. El modelo de asesoría y acompañamiento pedagógico implica el fortalecimiento de las habilidades docentes, por lo que el acompañamiento se concentra en las primeras implementaciones de la metodología, para dar paso progresivamente a la autonomía del docente una vez que se ha apropiado de la misma. 
Por otra parte, el programa $\mathrm{A}+\mathrm{S}$ ha generado diversas herramientas para apoyar la implementación de la metodología, entre las que se destaca la Guía de orientación para docentes (Castro, Moretti, Poblete y Reutter, 2007), la Rúbrica para evaluar la calidad del servicio (Casas Cordero, 2009) y el Modelo de sistematización para experiencias $A+S$ (Contreras, 2010).

Finalmente, en el modelo A+S UC la gestión de los socios comunitarios municipales se realiza por lo general en el Programa Puentes UC ${ }^{4}$ y, para el caso de las organizaciones de la sociedad civil, esta se verifica de forma directa por medio del profesor del curso o del encargado de carrera A+S. Es importante destacar aquí que la elección y definición del servicio por implementar requiere de algunas condiciones. En primera instancia, se debe tener en cuenta que los servicios siempre responden a una necesidad sentida de la comunidad $y$, en segundo término, estos tienden a la promoción social y no al asistencialismo, ya que están basados en una concepción de relación horizontal entre la universidad y el socio comunitario, quien es un agente activo en el diagnóstico, comprensión y resolución de sus problemáticas.

\section{Resultados}

En la actualidad cerca de 100 cursos de la UC se desarrollan anualmente con la metodología A+S y alrededor de 18.000 estudiantes han tenido un curso con esta metodología (Tabla 1), la que se ha implementado en cursos de la mayoría de las facultades de la UC (Gráfico 1).

Tabla 1: Coberturas de Aprendizaje Servicio UC: 2005 - 2013

\begin{tabular}{|l|c|r|r|r|r|r|r|r|c|}
\hline & 2005 & 2006 & 2007 & 2008 & 2009 & 2010 & 2011 & 2012 & $\begin{array}{c}2013 \\
\left(1^{\circ} \text { semestre }\right)\end{array}$ \\
\hline $\mathrm{N}^{\circ}$ cursos & 29 & 63 & 61 & 48 & 46 & 95 & 117 & 94 & 40 \\
\hline $\mathrm{N}^{\circ}$ profesores & 39 & 85 & 91 & 101 & 76 & 123 & 107 & 107 & 65 \\
\hline $\mathrm{N}^{\circ}$ estudiantes & 3.000 (estimado) & & 2.040 & 1.480 & 1.435 & 3.286 & 3.476 & 3.230 & 1.311 \\
\hline
\end{tabular}

4 Puentes UC es un programa del Centro de Políticas Públicas UC, cuyo fin es desarrollar iniciativas orientadas a la solución de problemas reales a nivel local. Más información en www.puentesuc.cl 
Gráfico 1: Cantidad de cursos implementados con A+S en la UC por Facultades 2006-2013

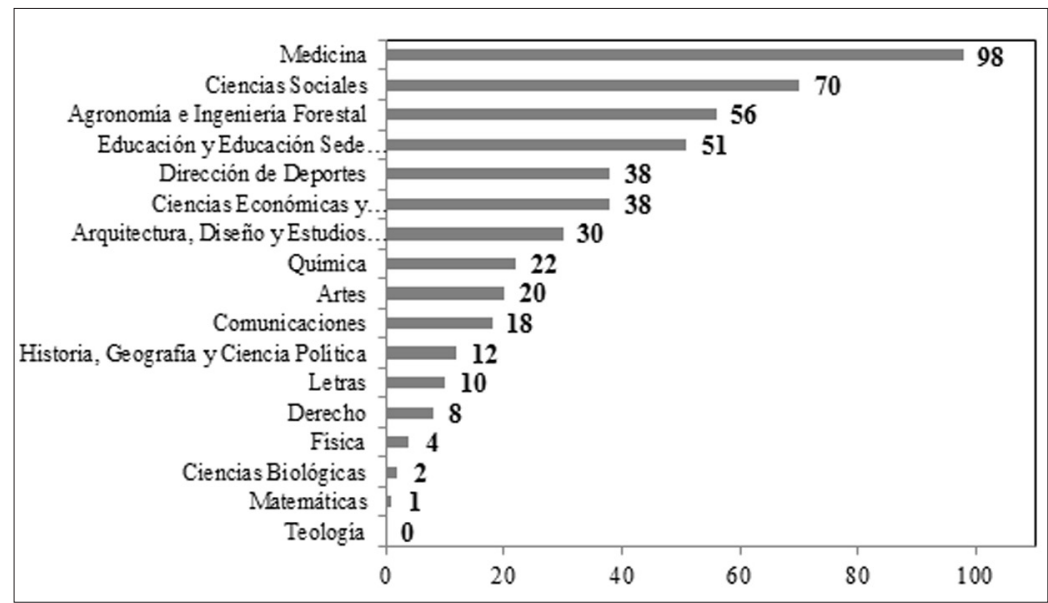

Una característica importante de la implementación de A+S en la UC tiene que ver con el desarrollo de acotados pero importantes estudios realizados por el mismo Programa, los que han sugerido líneas de acción y recomendaciones que han fortalecido al proyecto. Durante los primeros años (2004 - 2008) se realizaron estudios asociados a los procesos de implementación propiamente tal y a las dificultades y expectativas de los actores en torno al A+S. Desde el 2010 se han recogido datos para conocer y sintetizar los resultados que los diferentes actores atribuyen a $A+S$ sobre el aprendizaje de los estudiantes, las prácticas de los docentes y el desarrollo de las organizaciones socias. Algunos resultados que destacan son coherentes con las investigaciones existentes en la literatura internacional, entre los que se cuenta la mayor motivación por aprender, el desarrollo de habilidades interpersonales y el trabajo en equipo, el mejor dominio de competencias técnicas y el desarrollo de valores, entre otros.

Un estudio realizado en 2011 por Berríos, Contreras, Herrada, Robles y Rubio (2012) incorporó un análisis cuantitativo del cuestionario de evaluación de cursos y un análisis cualitativo de las respuestas abiertas de este, además de entrevistas a estudiantes en proceso de titulación que habían cursado entre tres y siete cursos con esta metodología. En este estudio, los estudiantes reportan 
que $\mathrm{A}+\mathrm{S}$ es una estrategia que ayuda a mejorar el aprendizaje en lo relativo al ejercicio profesional y en sus formas de aplicación, posibilitando la integración de diferentes saberes, los que pueden ser contenidos disciplinares, como también de manera muy importante, habilidades necesarias para ser un buen profesional, las habilidades comunicativas, la capacidad de resolver asuntos complejos o el trabajo en equipo. Igualmente allí se explicita que $\mathrm{A}+\mathrm{S}$ permite integrar el compromiso social en la propia disciplina, apareciendo con mucha fuerza consideraciones acerca de la responsabilidad social y personal de la profesión desarrollada. La sensación de estar realizando una acción que tendrá consecuencias positivas reales en sus socios, o por el contrario, que puede perjudicarlos, mejora en los estudiantes la percepción de sí mismos y, al mismo tiempo, el compromiso personal con su actividad profesional.

Destacan en dicho estudio los aspectos de la experiencia que posibilitarían esos aprendizajes, entre los que se puede mencionar la relación con los otros y la existencia de reflexión al interior del curso. Asociado a las relaciones establecidas en la experiencia, los estudiantes reportaron que el rol de sus profesores cambia, siendo tanto o más importantes que la transmisión de los contenidos la discusión con ellos y la guía que puedan prestarles en la actividad que ejecutan. La cercanía con el socio comunitario, por su parte, está totalmente integrada a la experiencia de aprendizaje y es fundamental para esta práctica. Por otra parte, los estudiantes indicaron que la existencia de reflexiones era un componente clave para el proceso de aprendizaje, porque permite conectar la experiencia de servicio con los contenidos del curso, otros saberes complementarios a la disciplina, así como habilidades o aspectos valóricos del ejercicio profesional.

Del mismo modo en 2010 y 2011 se investigó en torno a la percepción de los docentes respecto del impacto de A+S (Cisternas, 2010; Berríos et al., 2012). En estos estudios se constató que los profesores coinciden en atribuir a la metodología A+S la posibilidad de favorecer mejores resultados de aprendizaje, tanto de los contenidos y herramientas propios del curso como desde el punto de vista de la formación integral del ciudadano y futuro profesional que tienen a cargo. Esto posibilita que, aun cuando en su totalidad 
consideran que $\mathrm{A}+\mathrm{S}$ implica mayor esfuerzo que un curso normal y dan cuenta de algunas dificultades en la implementación, en general sean perseverantes en su implementación, o bien, deseen apoyar la proyección de la metodología como herramienta institucionalizada en las apuestas curriculares de la universidad.

Otro elemento por destacar de estas investigaciones es que los docentes perciben que la incorporación de A+S tiene un efecto en su práctica pedagógica, señalando principalmente el desarrollo de habilidades docentes necesarias para guiar estas experiencias y la posibilidad de integrar sus convicciones valóricas e intereses sociales en su docencia. Entre las herramientas pedagógicas desarrolladas se encuentran algunas técnicas de evaluación formativa, la planificación de cursos y clases, la facilitación de espacios de discusión y la actualización de los conocimientos respecto de los problemas sociales emergentes, entre otros aspectos. Los docentes también reportan que la relación con sus estudiantes mejora, en la medida que su rol comienza a orientarse al acompañamiento y la guía de sus estudiantes, en una labor de carácter cada vez más formativa, al tiempo que pueden integrar en su quehacer el servicio con la docencia, contribuyendo a una relación positiva entre academia y sociedad. Por último, los académicos señalaron algunos puntos críticos que dificultaron la implementación de la metodología, relacionados principalmente con la mayor carga de trabajo, la falta de reconocimiento y promoción del trabajo realizado y el poco apoyo general de parte de las unidades académicas y de la universidad.

Por su parte, la percepción de los socios comunitarios también ha sido recogida desde los primeros años de funcionamiento de $\mathrm{A}+\mathrm{S}$ UC mediante estudios y evaluaciones finales de la experiencia. A partir de esta información es posible establecer que los socios comunitarios se muestran satisfechos con los servicios recibidos, tanto de forma global en relación con la experiencia como en aspectos específicos relativos a los productos como la calidad o la utilidad del servicio. Los estudios realizados demuestran que los representantes de las organizaciones socias han evidenciado valoraciones positivas asociadas al apoyo experto de la universidad en sus áreas de trabajo, tanto de los estudiantes como de los docentes de los cursos, quienes entregan 
una mirada externa y profesional respecto de los problemas que enfrenta la organización. Al mismo tiempo, los servicios en general han sido valorados positivamente gracias a la utilidad que presentan para la organización y sus destinatarios, así como su capacidad para solucionar o aportar en la solución de necesidades sentidas por parte de las organizaciones.

\section{Conclusiones}

En la UC el modelo de implementación de $\mathrm{A}+\mathrm{S}$ ha permitido generar resultados positivos en la formación profesional integral de los estudiantes, la formación docente y el servicio a la comunidad. El desarrollo en los estudiantes de habilidades, actitudes y valores como el compromiso social, el trabajo en equipo y la resolución de problemas, posiciona a la metodología como una herramienta eficaz para la formación de profesionales competentes y comprometidos con la sociedad.

Estos resultados pueden relacionarse, al menos, con tres aspectos que han caracterizado el desarrollo del modelo en la UC. En primer término, la coherencia de la metodología $\mathrm{A}+\mathrm{S}$ con el Plan de desarrollo de la UC, su Proyecto educativo y las características del egresado expresadas en la Impronta UC, han permitido una instalación armónica del modelo en la institución; en segundo lugar, la incorporación del Programa A+S a la Vicerrectoría Académica ha facilitado la inserción desde una lógica curricular, permitiendo generar coherencia entre el modelo $\mathrm{A}+\mathrm{S}$ y la formación docente de la universidad; por último, la existencia de una reflexión constante en torno al proceso de implementación y sus resultados, tanto de los actores de cada curso como desde el equipo de profesionales del Programa, ha permitido que se configure como un modelo en constante desarrollo. Los resultados de evaluaciones e investigaciones, en esta línea, han sido un insumo importante en este ejercicio reflexivo.

Entre los desafíos y proyecciones del modelo actual se encuentra la necesidad de reconocer, de manera efectiva, las horas de trabajo docente que implica la incorporación de la metodología a un 
curso, así como también incluir algún mecanismo de reconocimiento de las buenas prácticas en este tipo de cursos por parte de la unidad académica o de la universidad. Por otro lado, se hace necesario revisar en las carreras la cantidad de los cursos A+S que los estudiantes realizan, de manera de distribuir equitativamente los cursos a lo largo del currículo.

\section{Referencias bibliográficas}

Berríos, V., Contreras, M., Herrada, M., Robles, M. y X. Rubio, (2012) Resultados de Aprendizaje Servicio en la UC: informe final de investigación. Santiago, Programa Aprendizaje Servicio, Pontificia Universidad Católica de Chile.

Casas-Cordero, M., (2009) Rúbrica para la calidad del servicio. Santiago, Programa Aprendizaje Servicio, Pontificia Universidad Católica de Chile.

Castro, C., Moretti, R., Poblete, X. y F. Reutter, (2007) Guías de orientación para docentes. Santiago, Programa Aprendizaje Servicio, Pontificia Universidad Católica de Chile.

Cisternas, P., (2010) Factores que inciden en la continuidad de los profesores en el programa A+S UC. Santiago, CDDoc, Pontificia Universidad Católica de Chile.

Contreras, M., (2010) Modelo de sistematización: una herramienta para sistematizar cursos que implementan Aprendizaje Servicio en la UC. Santiago, Programa Aprendizaje Servicio, Pontificia Universidad Católica de Chile.

Cooper, S., Cripps, J., y Reisman, J. (2013). "Service-learning in deaf studies: impact on the development of altruistic behaviors and social justice concern", en American annals of the deaf. 157(5), enero, pp. 413-27.

Eyler, J., (2001) "Creating your reflection map", en New directions for higher education, Issue 114, Summer, doi: 10.1002/he.11.

Eyler, J. (2009) "The power of experiential education", en Liberal Education, 95(4), otoño, pp. 24-31.

Furco, A. y S, Billig, (2002) Service learning: the essence of pedagogy. Connecticut, IAP.

Groh C., Stallwood L. y J, Daniels, (2011) "Service-learning in nursing education: its impact on leadership and social justice", en Nurs Educ Perspect. 32(6), Nov. Dic. pp. 400-5. 
Hansen, K., (2012) "A practical guide for designing a course with a ServiceLearning component in higher education", en The Journal of Faculty Development. 26 (1), pp. 29-36.

Howard, J., (2003) "Service Learning research: foundational issues", en Billig, S. y A. Waterman, (Eds.) Studying Service Learning: innovations in education research methodology. New Jersey, Lawrence Erlbaum Associates.

Jenkins, A. y P. Sheehey, (2011) "A checklist for implementing ServiceLearning in higher education", en Journal of Community Engagement and Scholarship. 4 (2), junio, pp. 52-60.

Newman, C. y S. Hernández, (2011). Minding our business: longitudinal effects of a service-learning experience on alumni. A Journal of College Teaching and Learning.; 8 (8), agosto, pp. 39-48.

Pontificia Universidad Católica de Chile, (2010) "Plan de Desarrollo 20102015". [En línea]. Santiago, disponible en: http://rectoria.uc.cl/index. php?option=com_docmanEtask $=$ doc_download $E$ gid $=75 \&$ Itemid $=65 \& l a$ $n g=e s$ [Accesado el 28 de agosto de 2013].

Tapia, M., (2006) La solidaridad como pedagogía. Buenos Aires, Ciudad Nueva. 